\title{
Simulation of Plasma Density Gradient Generation by Wakefield and the Effect of Probe Pulse Time-Delay on the Photon Acceleration
}

\author{
Marwa Mohammed Abd Elwahab1, Haron Abd Elfatah Elshaikh1, Ossama Mohammed Yassin² \\ ${ }^{1}$ Department of Physics, Aswan University, Aswan, Egypt \\ ${ }^{2}$ Department of Physics, Al-Azhar University, Cairo, Egypt \\ Email:d.marwaj@gmail.com
}

How to cite this paper: Abd Elwahab, M.M., Elfatah Elshaikh, H.A. and Yassin, O.M. (2021) Simulation of Plasma Density Gradient Generation by Wakefield and the Effect of Probe Pulse Time-Delay on the Photon Acceleration. Open Journal of Applied Sciences, 11, 354-364.

https://doi.org/10.4236/ojapps.2021.113026

Received: October 14, 2020

Accepted: March 28, 2021

Published: March 31, 2021

Copyright $\odot 2021$ by author(s) and Scientific Research Publishing Inc. This work is licensed under the Creative Commons Attribution International License (CC BY 4.0).

http://creativecommons.org/licenses/by/4.0/

\begin{abstract}
Laser Wakefield is produced by ultra-high intensity laser pulse interacting with underdense plasma with special conditions for the laser wavelength and plasma density. In this mechanism, nonlinear forces appear due to the very high amplitudes of the electromagnetic wave and these forces evacuate plasma electrons from the path of the laser pulse leading to very high electron plasma density gradients. Due to the electrostatic forces which result from these density perturbations, the electrons move very fast in oscillatory manner to restore neutrality creating a wake of electron density perturbations behind the laser pulse. Detailed investigation has been dealt with the time-delay between the driver laser pulse and the probe pulse which can affect the production of high plasma gradients needed for photon acceleration process.
\end{abstract}

\section{Keywords}

Plasma Wakefield, Photon Acceleration, Simulation

\section{Introduction}

High-intensity short-pulse laser-plasma interactions are of much current interest because of their applications [1] [2] [3]. When the intense laser or particle beam interacts with the plasma, it creates plasma waves. The generation of relativistic plasma waves by intense laser or electron beams in plasmas is important for producing ultra-high gradient. A number of methods are being pursued after the development of the laser/particle beam-matter interactions to achieve ultra-high 
gradients. These are including the laser Wakefield mechanism. The generation of the relativistic plasma waves using the laser Wakefield was first proposed by Tajima and Dawson in 1979 for producing ultra-high gradient plasma density. However, the intensity and the length of the pulse are controlling on the mechanism which, is used to generate the plasma waves. In the laser Wakefield the intensity is high $\left(I \simeq 10^{18}\right)$ with length ultra-short $\left(1.79874 \times 10^{-5}\right)$ [4]. In 1982 [5] Sullivan and Godfrey, and in 1984 [6] Mori simulated the LWAF in one-dimensional (1-D) particle-in-cell. Before 1988 the technology for generating ultra-intense picoseconds laser pulse didn't exist. The LWAF was reinvented independently by [7] Gorbunov and Kirsanov and [8] [9] Sprangle et al. In 1989 [10] Bulanov et al., also in 1990 Sprangle et al. [11] [12] and Berezhiani and Murusidze [13] developed the nonlinear theory of LWFA in one dimension. In 1992 [14], and in 1993 [15] Sprangle et al. analyzed the nonlinear theory of the LWFA in two dimensions including the self-consistent evolution of the laser pulse. In 1993 [16] Hamster et al. obtained the first experimental evidence for plasma wave generation by the LWFA mechanism. In 1999 [17], the ray tracing simulation was used to diagnose future plasma acceleration and it was found that the photon acceleration was the most appropriate diagnostic technique for the future laser Wakefield accelerators. In 2002 [18], the simulation of the photon acceleration was studied by the interaction between the ultra-short intense laser pulse and the gradient of plasma density, the frequency up shift was observed clearly in $1 \mathrm{~d}$ simulation and small in $2 \mathrm{~d}$ simulation. The saturation mechanisms and the effects of slippage, dispersion, diffraction and pump depletion for parameter optimization were discussed. Also in 2002 [19], the simulation of the photon acceleration was studied using plasma wake fields and the amplification of the laser pulse using Raman backscattering. The simulation of the photon acceleration and laser pulse amplification presented with electromagnetic PIC simulation codes, 1d-XOOPIC and 2d OSIRIS observed the frequency up shift photon acceleration simulation after $2 \mathrm{~cm}$ of pulse propagated up to $150 \%$ in $1 \mathrm{~d}$ simulation, but only $10 \%$ for $2 \mathrm{~d}$ simulation. In the simulation of laser pulse amplification, 1d fluid model was developed for the slowly varying envelope equations to investigate the amplification characteristics. The seed pulse is amplified exponentially with the growth rate in linear regime, and proportionally to $t_{1}$ in nonlinear regime. In 2004-2005 [20] [21], C. D. Murphy et al. studied experimentally and numerically, the photon acceleration by changed the gas density in experiment and changed the plasma density in the numerical studies with ultra-short laser pulse, the blue shift was observed and it decreased with increasing plasma density. In 2006 [22], R. Trines, et al. used the same parameter in the experimental and also in the simulation that it used ultra-short intense laser pulse with change in the plasma density, the photon acceleration was observed and agreement between the numerical and the experimental. Also in 2006 [23], A. J. W. Reitsma, et al. studied laser pulse propagation in underdense plasmas with two different one-dimensional codes for two laser pulse lengths that are relevant 
to the ongoing development of laser Wakefield acceleration, agreement between the full wave and photon kinetic approaches. In 2007-2008 [24], R. M. G. Trines et al. studied the role of the photon acceleration of a long laser pulse (180fs) with underdense plasma. They observe that the laser pulse modulated on the length scale of the laser-driven plasma wave and predicted that the plasma wave will also modulate the laser frequency. In 2012 [25], the frequency up-shift phenomena during the interaction of an intense laser pulse with helium gas target was observed. This shifted due to the Wakefield-induced modulation of the laser pulse and used PIC code on this propagation. In 2013 [26], the generation of longitudinal electrostatic Wakefield was studied by interaction of super-Gaussian laser pulse with homogeneous plasma presented by 2D PIC simulation in the relativistic regime. It is observed that the generated wake amplitude for super-Gaussian driving laser pulse increase as compared to the Wakefield amplitude for a Gaussian laser pulse. By developing a 1D numerical model the Wakefields generated due to super-Gaussian laser pulse are then compared to the wakes driven by Gaussian laser pulse. The results obtained via numerical studies and simulations are found to be in good agreement with each other. In 2015 [27], the photon acceleration was shown as a particular case of the inelastic scattering process, where a probe photon increased, their energy from a moving in density perturbated. This observation illustrated by studying the interaction of a probe photon beam with bubble which produced by intense laser pulse. Still in 2015 [28], sending a long laser probe pulse behind the short pump pulse, generates the Wakefield. By this simulation the measurement of a density profile in a plasma Wakefield can be performed using the photon acceleration.

In this research, the acceleration of the probe pulse photons was studied by generating a Wakefield behind a short-duration ultra-high-intensity pump pulse propagate in underdense density plasma that was calculated from the wavelength of the laser pulse, compared to the acceleration produced in a reference [18] which used a high-intensity pulse and also reference [11] with changed on density of the plasma. He found a very high acceleration of the probe's pulse photons. The effect of the time delay change between the pulses generated the Wakefield and that will accelerate and the extent of the effect of this change on acceleration was also studied. This change was compared to reference [29] where the study was carried out for the time delay values were calculated from the wavelength of the plasma wave, found that the probe pulse is down shifted (photon deceleration) or up shifted (photon acceleration) unlike what was calculated in our current research where the time delay values were calculated relative to the wavelength of the pump pulse and found that all times have the same effect on acceleration.

In Section 2, introduction to photon acceleration and the equation used in this acceleration. In Section 3, the conditions generate the Wakefield used in this acceleration. In Section 4, the simulation used to generate the Wakefield and calculate the time delay then the results from this simulation. Finally is the conclu- 
sion.

\section{Photon Acceleration}

The concept of photon acceleration can be used to describe the spectral changes experienced by electromagnetic waves when they propagate in spatially and temporally varying plasmas. When the space and time scales of the plasma perturbations are much larger than the photon wavelength and period, geometric optics can be used to describe the motion of the electromagnetic wave-packets. Photons can exchange energy with the plasma through the action of the ponderomotive force. The upshift frequency of an electromagnetic wave propagating through plasmas is [18]

$$
\frac{\Delta \omega}{\omega_{0}}=-\pi \frac{\cos \left(k_{p 0} \zeta\right) \omega_{p 0}^{2} \nu_{g} \tau}{n_{0} \lambda_{p 0}}\left(\frac{\delta n_{0}}{n_{0}}\right)
$$

where $v_{g}$ and $\tau$ are the group velocity of the probe pulse and the propagation time, respectively. $\omega_{p 0}$ is the electron plasma frequency characterized by the characteristic dispersion relation [30]

$$
\omega_{p 0}^{2}=\omega_{P}^{2}+3 k^{2} \vartheta_{t h e}^{2}
$$

where $\omega$ and $k$ are the frequency and wavenumber of the electrostatic oscillations respectively, and $\vartheta_{\text {the }}=\sqrt{T_{e} / m}$ is the electron thermal velocity and $T_{e}$ the electron temperature [31].

An electron plasma wave is considered one of the basic types of electrostatic waves, because its frequency is nearly equal to the electron plasma frequency. [32].

Some mechanisms can be used to generate perturbation in the plasma density. In this work the density gradient generation by Wakefield is thoroughly investigated.

\section{Wakefield Plasma Density Gradient Generation}

When a short laser pulse with frequency is much larger than the plasma frequency and due to the ponderomotive force, an electrostatic wake will be excited [33], as in Figure 1. An intense pulse with a group velocity [34].

$$
v_{g}^{E M}=c\left(1-\omega_{p}^{2} / \omega_{0}^{2}\right)^{\frac{1}{2}}<c
$$

Propagate through underdense plasma $\left(\omega_{0} \gg \omega_{P}\right)$, the ponderomotive force expels electrons from the region of the laser pulse and excites electrons gradient. [35]

$$
F_{\text {pond }} \cong-(m / 2) \nabla v_{\text {osc }}^{2}
$$

These waves are generated as a result of being displaced by the leading edge of the laser pulse. If the laser pulse length $L=c \tau_{l}$ is larger than the electron 


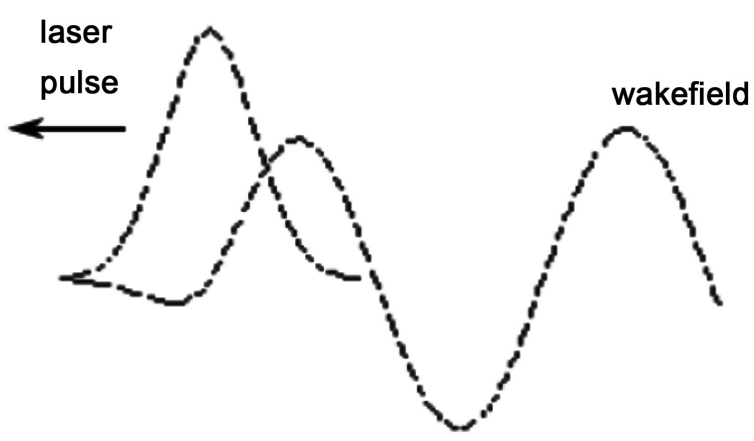

Figure 1. A short laser pulse propagating in a plasma excites a relativistic electron plasma wave.

plasma wavelength (where $\tau_{l}$ is the pulse duration), $\lambda_{p}=\frac{2 \pi c}{\omega_{p}}$ [36] the energy in the plasma wave is re-absorbed by the trailing part of the laser pulse. However, if the pulse length is approximately equal to or shorter than the plasma wavelength $c \tau_{l}<\lambda_{p}$ [37] [38], the ponderomotive force excites plasma waves or Wakefields with phase velocity equal to the laser group velocity and the energy is not re-absorbed.

\section{Simulation Results}

Simulation experiments with different probe pulse parameter are conducted to observe the effect of the variation of this parameter on the nature of the interaction of this pulse with the Wakefield, and how this affects the upshift of the frequency of the photons in the probe pulse.

The time delay is selected with respect to the ratio $\left(\frac{\lambda}{c}\right)$ for the pump pulse. The time of the wavelength for the pump pulse is calculated, and then the selection of the time delay for the probe pulse is done according to the calculated time of the pump pulse ( $t=\frac{\lambda}{c}$ ) as equals to $0.0 t, 0.25 t, 0.5 t, 0.75 t$ and $t$.

Figures 2(a)-(d) show the phase space of electrons (perturbation of plasma density) after generation. The amplitude of density gradient produced from the propagation of the pump pulse in the plasma with ultra-high intensity $I=7.04 \times$ $10^{19} \mathrm{~W} / \mathrm{cm}^{2}$ and ultra-short duration $60.0 \times 10^{-15} \mathrm{~s}$ was in the range of the expected value.

Figures 3(a)-(d) show the 3-D representation of the simulation results of frequency upshift for the probe pulse propagation at different simulation times. The probe pulse is sent after the pump pulse with times delay of $\left[0.0,0.25 \frac{\lambda}{\mathrm{C}}\right.$, $\left.0.5 \frac{\lambda}{c}, 0.75 \frac{\lambda}{c}, 0.75 \frac{\lambda}{c}\right]$ with the same wavelength but with a lower duration, $14.9895 \times 10^{-15} \mathrm{~s}$. The intensity of the probe pulse is much lower than the pump pulse $\simeq 8.98 \times 10^{14} \mathrm{~W} / \mathrm{cm}^{2}$. Photons can exchange energy with the density gradient of the plasma through the action of the ponderomotive force. The 


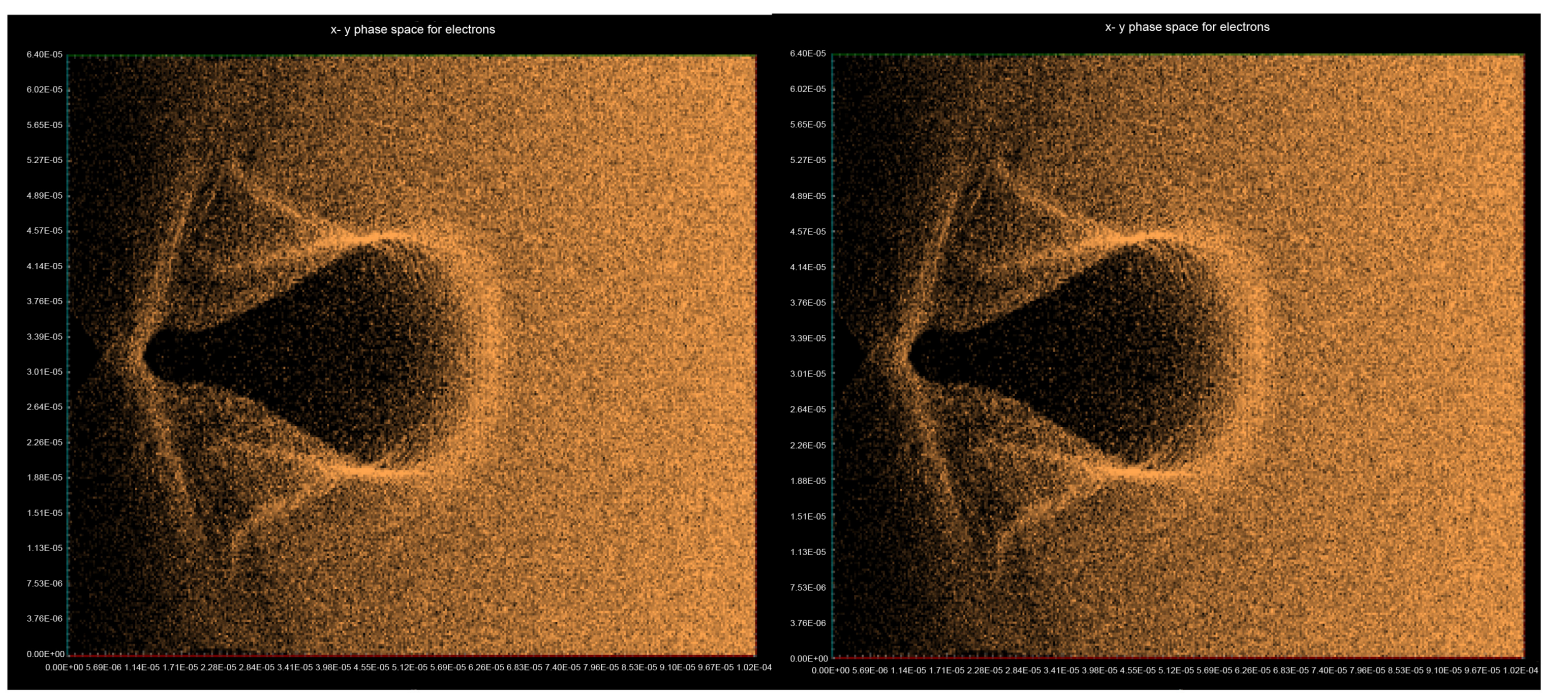

(a)

(b)

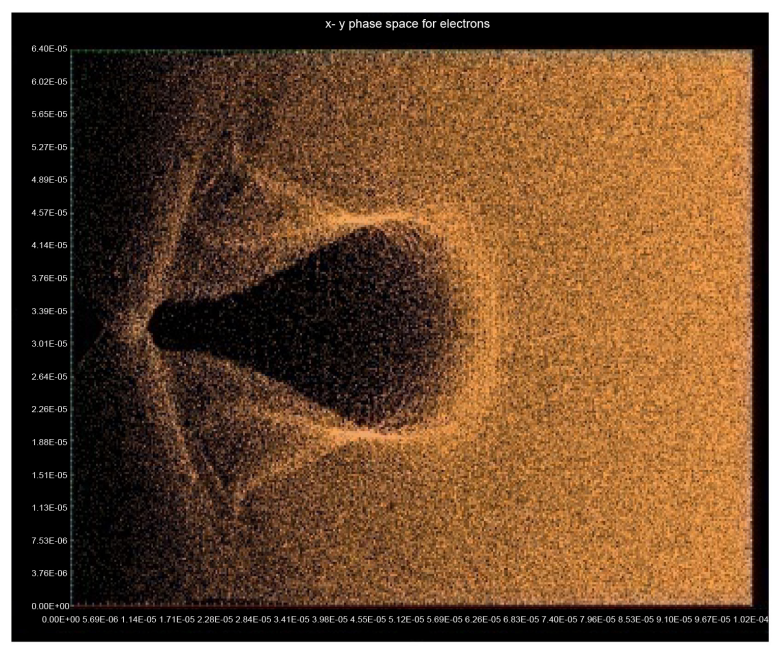

(c)

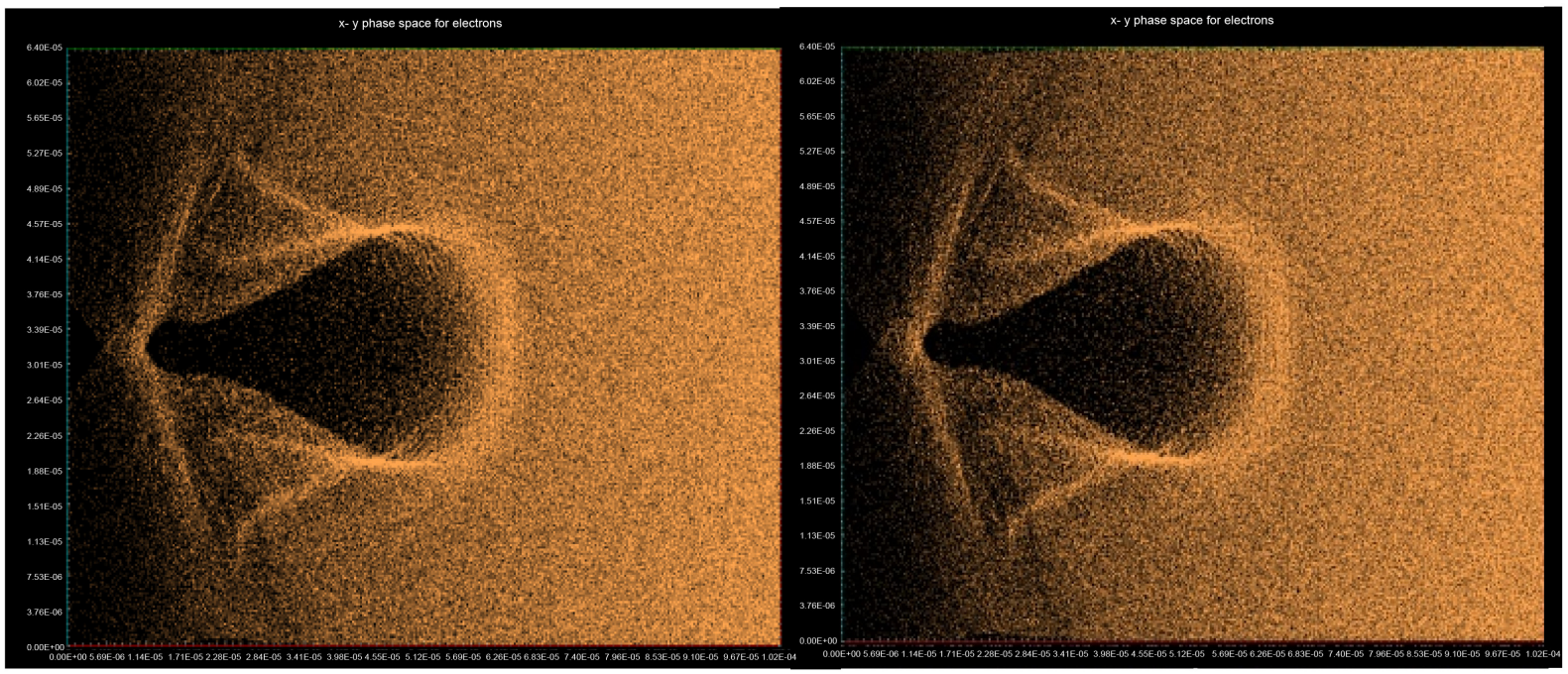

Figure 2. Phase space electrons of times delay $\left[0.0,0.25 \frac{\lambda}{c}, 0.5 \frac{\lambda}{c}, 0.75 \frac{\lambda}{c}, \frac{\lambda}{c}\right]$ respectively at simulation time $2.00110 \times 10^{-13}$ s. 


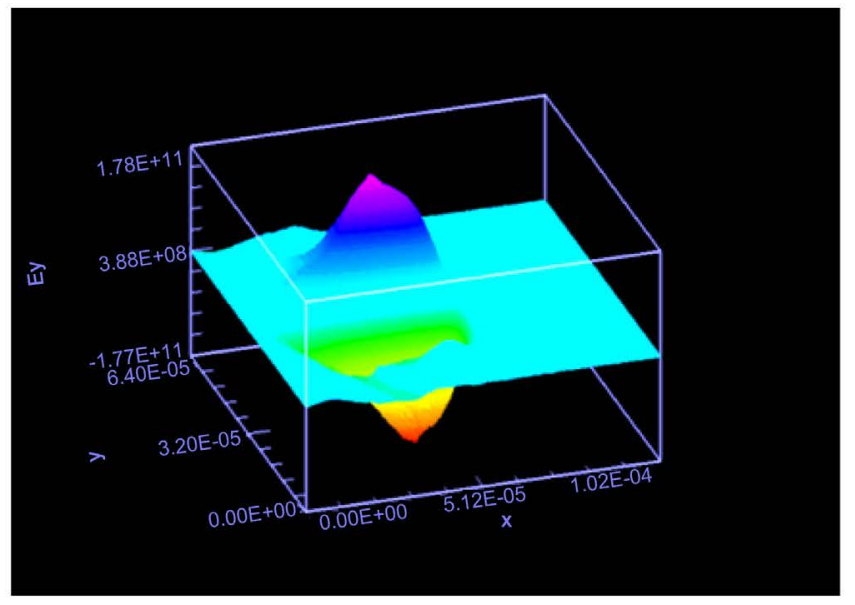

(a)

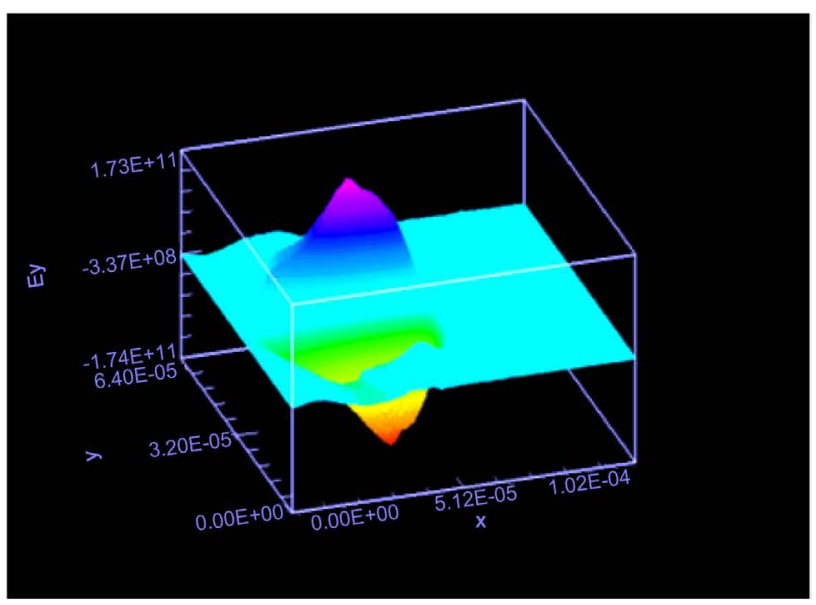

(b)

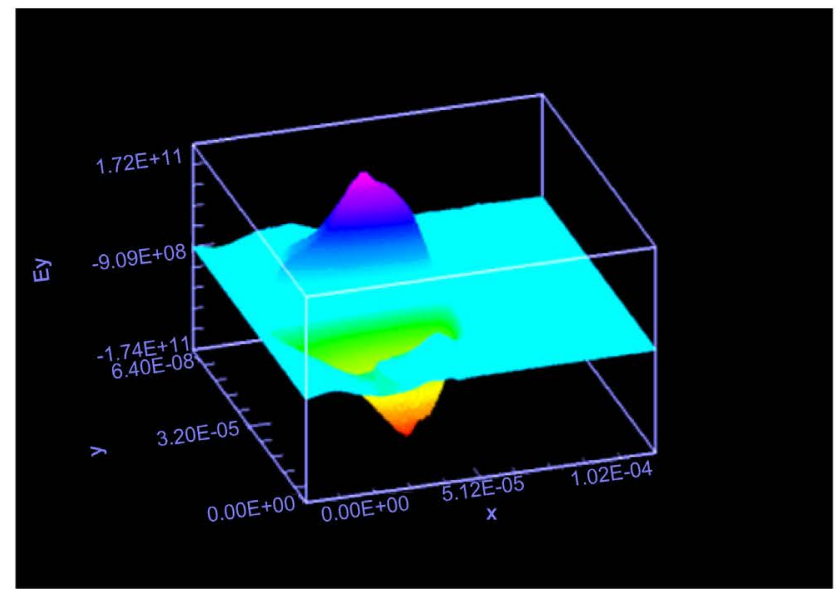

(c)

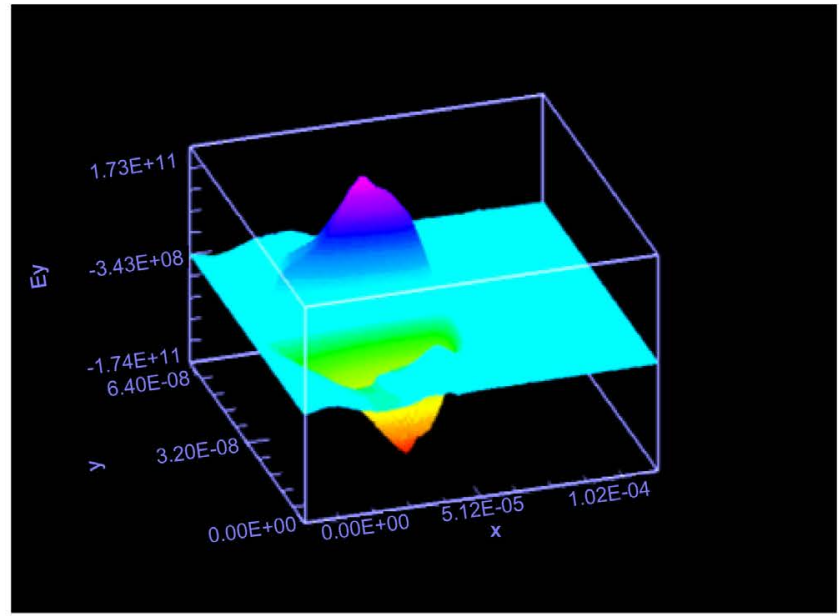

(d)

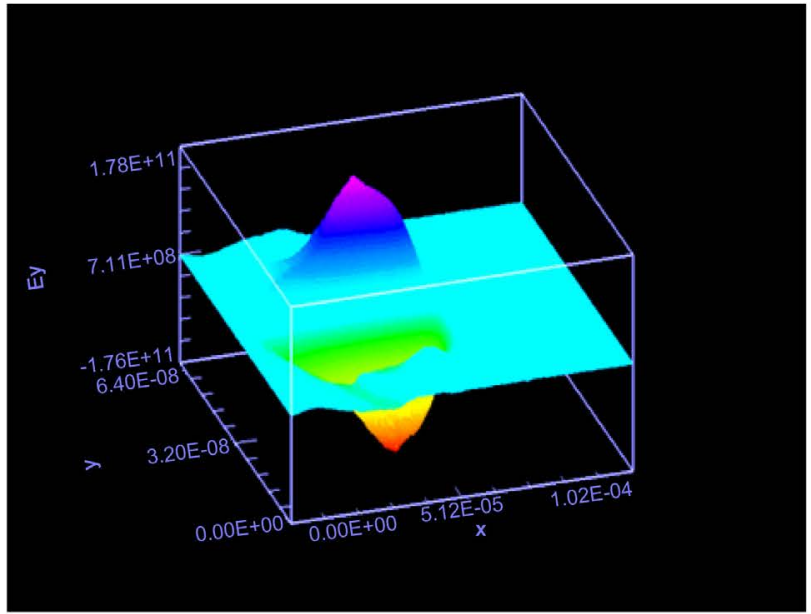

(e)

Figure 3. The 3-D graph of the probe pulse of times delay $\left[0.0,0.25 \frac{\lambda}{c}, 0.5 \frac{\lambda}{c}, 0.75 \frac{\lambda}{c}, \frac{\lambda}{c}\right]$ respectively at simulation time $2.00110 \times 10^{-13} \mathrm{~s}$.

upshift of the photons continues to a greater extent at later times. Positive frequency shifts (upshifted) require the laser probe pulse to be centered about 
regions of the wave with a decreasing density as shown in Figure 4. The comparison between the electric field of the probe pulse at various times delay is shown in Figure 5.

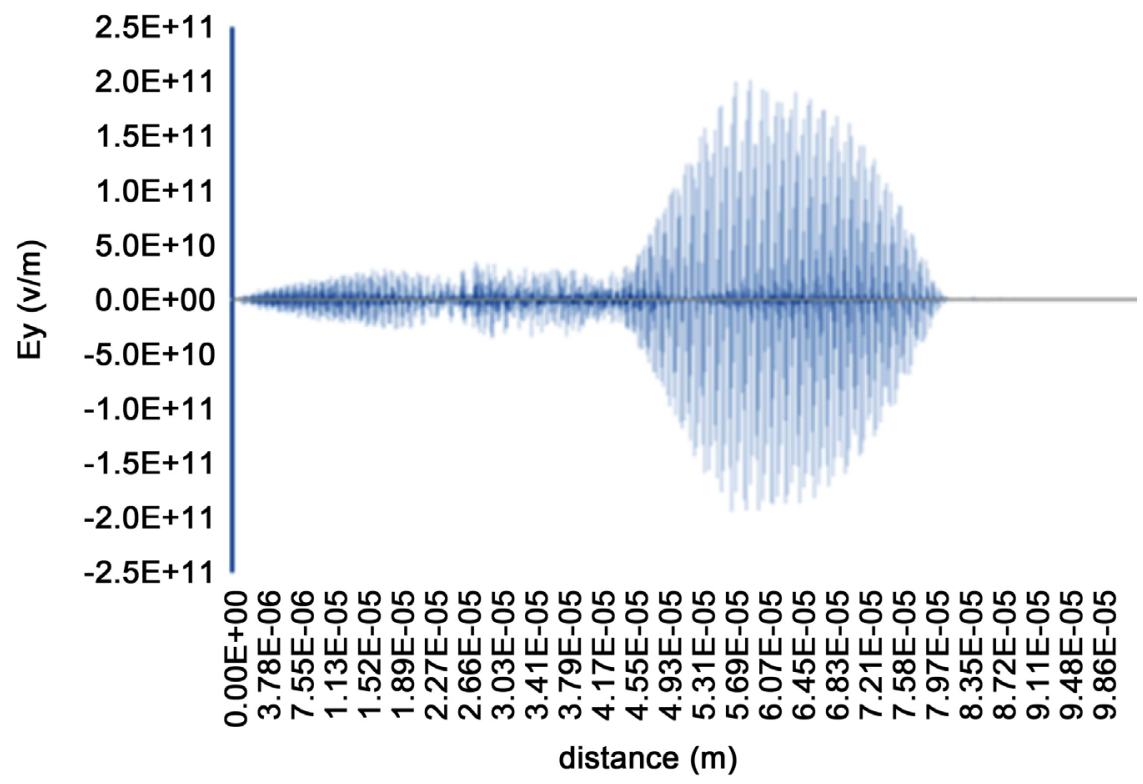

Figure4. Diagram of probe pulse upshifted.

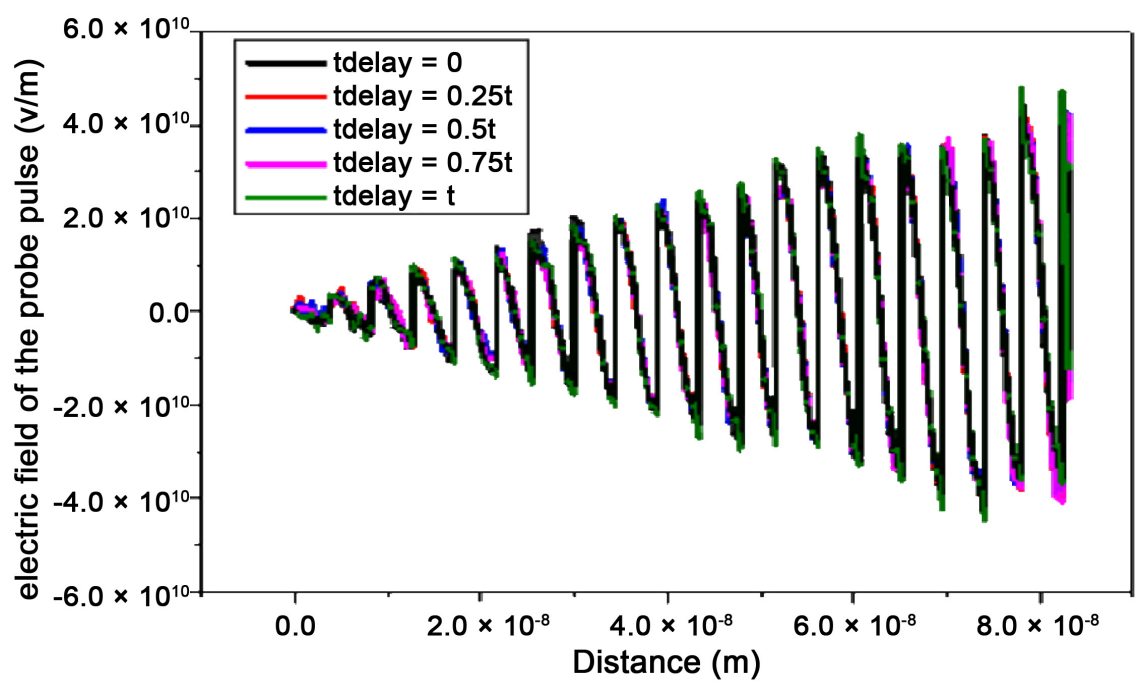

Figure 5. The electric field amplitudes of the probe pulse a function of distance at various time delays.

\section{Conclusion}

Equation (1) with its components gives the relation between the frequency shift $\frac{\Delta \omega}{\omega_{0}}$ and the density perturbation $\frac{\delta n_{0}}{n_{0}}$. The results have been obtained to demonstrate this relation. A single ultra-high intensity laser pulse when propagating in underdense plasma with special density calculated from the laser pulse 
wavelength, excites an electron plasma perturbation with very high gradient migrates following and behind the pulse and creates Wakefield with very high electrostatic amplitude. The results obtained in this paper for this case show qualitatively the effect of changing the time when the photons in the probe pulse interact with the electron plasma gradient in the wake of the drive pulse. According to the theory, as soon as this interaction happens, the photons will be upshifted.

\section{Conflicts of Interest}

The authors declare no conflicts of interest regarding the publication of this paper.

\section{References}

[1] Tabak, M., Hammer, J., Glinsky, M.E., Kruer, W.L., et al. (1994) Ignition and High Gain with Ultrapowerful Lasers. Physics of Plasmas, 1, 1626-1634. https://doi.org/10.1063/1.870664

[2] Amendt, P., et al. (1991) X-Ray Lasing by Optical-Field-Induced Ionization. Physical Review Letters, 66, 2589-2592. https://doi.org/10.1103/PhysRevLett.66.2589

[3] Umstadter, D. and Norris, T.B. (1997) Nonlinear Optics with Relativistic Electrons [Guest Editorial]. IEEE Journal of Quantum Electronics, 33, 1877-1878. https://doi.org/10.1109/JQE.1997.641304

[4] Bingham, R., Mendonca, J.T. and Shukla, P.K. (2004) Plasma Based Charged-Particle Accelerators. Plasma Physics and Controlled Fusion, 46, R1. https://doi.org/10.1088/0741-3335/46/1/R01

[5] Sullivan, D.J. and Godfrey, B.B. (1982) The Plasma Beat Wave Accelerator-11 Simulations. Proceedings of AIP Conference Laser Acceleration Particles, Vol. 91, 43-68.

[6] Mori, W.B. (1984) Computer Simulations on the Acceleration of Electrons by Fast Large-Amplitude Plasma Waves Driven by Laser Beams. Master's Thesis, University of California, Los Angeles.

[7] Gorbnnov, L.M. and Orsanov, V.I. (1987) Excitation of Plasma Waves by an Electromagnetic Wave Packet. Zhurnal Eksperimental noi i Teoreticheskoi Fiziki, 93, 509-518.

[8] Sprangle, P., Esarey, E., Ting, A. and Joyce, G. (1988) Laser Wakefield Acceleration and Relativistic Optical Guiding. Applied Physics Letters, 53, 2146-2148.

https://doi.org/10.1063/1.100300

[9] Esarey, E., Ting, A., Sprangle, P. and Joyce, G. (1980) The Laser Wakefield Accelerator. Comments on Plasma Physics and Controlled Fusion, 12, 5-3727.

[10] Bulanov, S.V., Kirsanov, V.I. and Sakharov, A.S. (1989) Excitation of Ultra Relativistic Plasma Waves by Pulse of Electromagnetic Radiation. JETP Letters, 50, 198-201.

[11] Sprangle, P., Esarey, E. and Ting, A. (1990) Nonlinear Theory of Intense Laser Plasma Interactions. Physical Review Letters, 64, 2011-2014. https://doi.org/10.1103/PhysRevLett.64.2011

[12] Ting, A., Esarey, E. and Sprangle, P. (1990) Nonlinear Wakefield Generation and Relativistic Focusing of Intense Laser Pulses in Plasmas. Physics of Fluids B: Plasma Physics, 2, 1390-1394. https://doi.org/10.1063/1.859561

[13] Berezhiani, V.I. and Murusjdze, I.G. (1990) Relativistic Wakefield Generation by an Intense Laser Pulse in a Plasma. Physics Letters A, 148, 338-340. 
https://doi.org/10.1016/0375-9601(90)90813-4

[14] Sprangle, P., Esarey, E., Krall, J. and Joyce, G. (1992) Propagation and Guiding of Intense Laser Pulses in Plasmas. Physical Review Letters, 69, 2200-2203.

https://doi.org/10.1103/PhysRevLett.69.2200

[15] Esarey, E., Sprangle, P., Krall, J., Ting, A. and Joyce, G. (1993) Optically Guided Laser Wakefield Acceleration. Physics of Fluids B, 5, 2690-2697. https://doi.org/10.1063/1.860707

[16] Hamster, H., Sullivan, A., Gordon, S., White, W. and Falcone, R.W. (1993) Subpicosecond, Electromagnetic Pulses from Intense Laser-Plasma Interaction. Physical Review Letters, 71, 2725-2728. https://doi.org/10.1103/PhysRevLett.71.2725

[17] Dias, J.M., Lopes, N., Figueira, G. and Mendonc, J.T. (1999) Photon Acceleration as the Laser Wakefield Diagnostic for Future Plasma Accelerators. IEEE Particle Accelerator Conference (PAC 99), New York, 29 March-2 April 1999, 3725-3727.

[18] Lee, H.J., et al. (2002) Simulations of Photon Acceleration and Laser Pulse Amplification Using Laser Plasma Interactions. Proceedings of LINAC, Gyeongju, Korea.

[19] Albert, F., et al. (2014) Laser Wakefield Accelerator Based Light Sources: Potential Applications and Requirements. Plasma Physics and Controlled Fusion, 56, Article ID: 084015. https://doi.org/10.1088/0741-3335/56/8/084015

[20] Murphy, C.D., Trines, R.M.G.M., Collier, J.L., et al. (2004-2005) CLF Annual Report. 80-83.

[21] Murphy, C.D., Bingham, R., et al. (2004-2005) CLF Annual Report. 85-86.

[22] Trines, R., et al. (2006) Evidence of Photon Acceleration by Laser Wake Fields. Physics of Plasmas, 13, Article ID: 033108. https://doi.org/10.1063/1.2398927

[23] Reitsma, A.J.W., et al. (2006) Photon Kinetic Modeling of Laser Pulse Propagation in Underdense Plasma. Physics of Plasmas, 13, Article ID: 113104. https://doi.org/10.1063/1.2366577

[24] Trines, R.M.G., et al. (2007-2008) CLF Annual Report. 138-141.

[25] Pathak, N.C., et al. (2012) Frequency Shift of an Intense Laser Pulse Induced by Plasma Wave. Nuclear Instruments and Methods in Physics Research A, 680, 103-107. https://doi.org/10.1016/j.nima.2012.04.012

[26] Jha, P., et al. (2013) Wakefield Generation and Electron Acceleration by Intense Super-Gaussian Laser Pulses Propagating in Plasma. Laser and Particle Beams, 31, 6.

[27] Kasim, M.F., et al. (2015) Simulation of Density Measurements in Plasma Wakefields Using Photon Acceleration. Physical Review Special Topics-Accelerators and Beam, 18, Article ID: 032801.

[28] Dawson, J.M. and Oberman, C.R. (1963) Effect of Ion Correlations on High-Frequency Plasma Conductivity. Physics of Fluids, 6, 394. https://doi.org/10.1063/1.1706745

[29] Krishnagopal, S., et al. (2016) Simulation of Electron Acceleration by Two Laser Pulses Propagation in a Homogenous Plasma. Proceedings of IPAC10, Kyoto, 4059-4061.

[30] Schluck, F. (2016) Amplification of Ultra-Short Relativistic Laser Pulses by Stimulated Brillouin Scattering.

[31] Mendonca, J.T. (2001) Theory of Photon Acceleration.

[32] Nicholson, D.R. (1983) Introduction to Plasma Physics. Wiley, New York.

[33] Rax, J.M. and Fisch, N.J. (1993) Ultrahigh Intensity Laser-Plasma Interaction: A Lagrangian Approach. Physics of Fluids B, 5, 2578. https://doi.org/10.1063/1.860693 
[34] Wilks, S.C., et al. (1989) Photon Accelerator. Physical Review Letters, 62, 2600-2603. https://doi.org/10.1103/PhysRevLett.62.2600

[35] Bingham, R. (2006) Basic Concepts in Plasma Accelerators. Philosophical Transactions of the Royal Society, 364, 559-575. https://doi.org/10.1098/rsta.2005.1722

[36] Pukhov, A., et al. (2002) Laser Wake Field Acceleration: The Highly Non-Linear Broken-Wave Regime. Applied Physics B, 74, 355-361. https://doi.org/10.1007/s003400200795

[37] Liesfeld, B. (1977) A Photon Collider at Relativistic Intensity.

[38] Joshi, C. (2017) Laser-Driven Plasma Accelerators Operating in the Self-Guided, Blowout Regime. IEEE Transactions on Plasma Science, 45, 3134-3146. https://doi.org/10.1109/TPS.2017.2769455 